\title{
Measurements of Turbulence near the Sea Bed in a Tidal Current ${ }^{1}$
}

\author{
K. F. BOWDEN \\ Oceanography Department, University of Liverpool \\ Liverpool, England
}

\begin{abstract}
The turbulent fluctuations of velocity have been measured, using an electromagnetic flowmeter, at heights up to $\mathbf{1 . 7 5}$ meters above the sea bed. The area of observations was off Anglesey, North Wales, in depths of 12 to 22 meters, on a firm, sandy bottom and during neutrally stable conditions. A number of records of the longitudinal $(u)$ and vertical $(w)$ components of velocity were obtained, and a few records included the lateral $(v)$ component. From these the intensities, autocorrelations, and cross correlations for various time lags were computed and the corresponding $u, v, w$, and $u w$ spectra were determined. Sufficient numbers of records have been obtained to enable mean values to be derived for a given set of physical conditions.
\end{abstract}

\section{METHOD OF OBSERVATION}

In the series of observations described below, the turbulent fluctuations in the velocity of the flow of water were recorded with an electromagnetic flowmeter. A brief description of this instrument has been given by Bowden and Fairbairn [1956]. The measuring unit has an over-all diameter of $10 \mathrm{~cm}$, and the magnetic field is produced by a circular coil through which an alternating current at $50 \mathrm{cps}$ is passed. Two pairs of electrodes mounted on this unit enable the potential gradients due to two components of the flow of water past the unit to be measured. With the plane of the coil vertical, the longitudinal component $u$ and the vertical component $w$ of the flow may be recorded. By mounting the unit with the plane of the coil horizontal, it is possible to record the transverse component $v$ with the longitudinal component $u$. Using two units, the corresponding component, $u$ for example, at two points may be recorded simultaneously. The over-all response of the equipment was flat for periods greater than 1 sec, falling to 50 per cent at a period of 0.25 sec. When the flowmeter was used at sea, the measuring units were mounted on a tripod laid

1 Based on a paper presented at the International Symposium on Fundamental Problems in Turbulence and Their Relation to Geophysics sponsored by the International Union of Geodesy and Geophysics and the International Union of Theoretical and Applied Mechanics, held September 4-9, 1961, in Marseilles, France. on the sea bed. A triangular fin kept the units aligned to the mean flow while the tripod was being lowered.

Bowden and Fairbairn [1956] gave an account of a number of records obtained with this equipment in Red Wharf Bay, off Anglesey, North Wales, in 1954. Since then a considerably larger number of records covering a wider range of conditions has been obtained during further observations in the same area. This area was considered suitable, as the bottom is fairly flat and is composed mainly of firm sand. The tidal currents are approximately rectilinear, and near the bottom they reach velocities up to about $50 \mathrm{~cm} / \mathrm{sec}$.

Since the recording was limited to two channels, in addition to the time trace, only two components could be recorded simultaneously. By using two measuring units and suitably switching the inputs to the amplifiers, the two channels could be used for making a variety of recordings: $u$ and $w$, or $u$ and $v$, at one position; $u$ at two positions with a given vertical or transverse separation; or $w$ at two positions in a similar way. The height of a measuring unit varied from 50 to $175 \mathrm{~cm}$ above the bottom, giving vertical separations between the two units up to $125 \mathrm{~cm}$.

\section{ANaLYsis of Reconds}

Usually the duration of an individual record was $10 \mathrm{~min}$; the first stage in the analysis was to read the two traces on the record at 1-sec 
intervals. From these numerical data, the rootmean-square values, autocorrelations, and cross correlations for various time lags were computed. The energy spectra were derived from the correlation curves by carrying out the Fourier transformations numerically. Much of the calculation was performed by electronic computer, in the earlier stages at Manchester University and more recently on the 'Deuce' at Liverpool.

\section{Results}

When the intensities of the $u$ and $w$ components of turbulence were computed, or the autocorrelation and cross-correlation curves were plotted, for individual 10-min records, they showed large variations from one record to another. By taking the mean values for a group of about half a dozen records, however, the results were considerably more consistent. In the following discussion, the results of taking the mean values of each group of records, obtained under similar conditions, are considered.

$u, w$ records. Table 1 gives the mean values tion of the record. $r_{w w}$ is the coefficient of correlation between simultaneous values of $u$ and $w . L_{w}$ and $L_{w}$ represent the integral scales of the $u$ and $w$ fluctuations, respectively, obtained from the autocorrelation curves. $L_{u}$ and $L_{v}$ are defined by

$$
L_{u}=\int_{0}^{\infty} r_{u}(x) d x \quad L_{w}=\int_{0}^{\infty} r_{w}(x) d x
$$

where $r_{u}(x)$ represents the coefficient of correlation between values of $u$ with a time lag $\tau$ and $x=U_{\tau}$.

There is no clearly significant variation with height in any of the quantities $\left[u^{2}\right]^{1 / 2} / U,\left[w^{2}\right]^{1 / 2} /$ $U,[u w] / U^{2}$, or $r_{w w}$ in the range 50 to $175 \mathrm{~cm}$ above the bottom. The mean values, irrespective of height, and their standard errors are given in the last line of the table. The values in the table suggest that both $L_{u}$ and $L_{w}$ increase with height but $L_{v}$ more rapidly than $L_{u}$. From the regression equations, however, it was found that the increase in $L_{u}$ was insignificant, and the increase in $L_{w}$ was significant only at the 7 per cent level.

TABLE 1. Mean Values for Groups of $u, w$ Records

\begin{tabular}{|c|c|c|c|c|c|c|c|}
\hline $\begin{array}{l}\text { Height, } \\
\text { cm }\end{array}$ & $\begin{array}{l}\text { No. of } \\
\text { Records }\end{array}$ & $\frac{\left[u^{2}\right]^{1 / 2}}{U}$ & $\frac{\left[w^{2}\right]^{1 / 2}}{U}$ & $\frac{[u w]}{U^{2}} \times 10^{-\mathrm{a}}$ & $r_{u w}$ & $\begin{array}{c}L_{u} \\
\text { meters }\end{array}$ & $\begin{array}{c}L_{w} \\
\text { meters }\end{array}$ \\
\hline $\begin{array}{l}50,60 \\
75 \\
100,125 \\
150,175 \\
\text { All heights }\end{array}$ & $\begin{array}{r}6 \\
6 \\
5 \\
8 \\
25\end{array}$ & $\begin{array}{c}0.140 \\
0.142 \\
0.119 \\
0.123 \\
0.131 \pm 0.005\end{array}$ & $\begin{array}{c}0.064 \\
0.066 \\
0.068 \\
0.065 \\
0.066 \pm 0.002\end{array}$ & $\begin{array}{c}3.31 \\
3.20 \\
2.69 \\
3.52 \\
3.23 \pm 0.20\end{array}$ & $\begin{array}{c}-0.37 \\
-0.36 \\
-0.39 \\
-0.41 \\
-0.38 \pm 0.02\end{array}$ & $\begin{array}{l}3.16 \\
3.59 \\
3.27 \\
4.27 \\
3.57\end{array}$ & $\begin{array}{l}0.88 \\
1.25 \\
1.33 \\
1.56 \\
1.25\end{array}$ \\
\hline
\end{tabular}

of various quantities for four groups of records of the longitudinal component $u$ and the vertical component $w$ of the turbulent velocity at different heights above the bottom. The mean current is denoted by $U$. The notation $\left[u^{2}\right]$ is used to denote the mean value of $u^{2}$ over the dura- $u, v$ records. Nine records were obtained of the longitudinal component $u$ and the lateral component $v$ taken simultaneously. These were at four different heights, but the scatter between individual records was considerable, and no systematic variation with height was appar-

TABLE 2. Mean Values for $u, v$ Records

\begin{tabular}{lccccccc}
\hline $\begin{array}{c}\text { Height, } \\
\mathrm{cm}\end{array}$ & $\begin{array}{c}\text { No. of } \\
\text { Records }\end{array}$ & $\frac{\left[u^{2}\right]^{1 / 2}}{U}$ & $\frac{\left[v^{2}\right]^{1 / 2}}{U}$ & $\frac{[u v]}{U^{2}} \times 10^{-3}$ & $r_{u v}$ & $\begin{array}{c}L_{u}, \\
\text { meters }\end{array}$ & $\begin{array}{c}L_{v}, \\
\text { meters }\end{array}$ \\
\hline $\begin{array}{l}60,75,100, \\
125\end{array}$ & 9 & $0.110 \pm 0.008$ & $0.082 \pm 0.006$ & $1.52 \pm 0.53$ & $0.14 \pm 0.05$ & 3.68 & 1.60 \\
\hline
\end{tabular}


ent, so that in Table 2 they are treated as a single group.

The mean value of $\left[v^{2}\right]^{1 / 2} / U$ is intermediate between the values of $\left[w^{2}\right]^{1 / 2} / U$ and $\left[u^{2}\right]^{1 / 2} / U$. In fact, from all the records, $\left[w^{2}\right]^{1 / 2} /\left[u^{2}\right]^{1 / 2}$ averaged 0.51 and $\left[v^{2}\right]^{1 / 2} /\left[u^{2}\right]^{1 / 9}$ averaged 0.76 . The correlation between $u$ and $v$ is considerably lower than between $u$ and $w$, as would be expected. $r_{u v}$ does appear to differ significantly from zero, however, but no physical explanation could be found for this.

Spectra of $u, v, w$, and $u w$. Figure 1 shows the mean autocorrelation curves for $u$ and $w$ from the group of $6 u, w$ records at $75 \mathrm{~cm}$ above the bottom. At the largest values of $x$ for which $r_{u}(x)$ and $r_{v}(x)$ could be computed, the values of the coefficients were still appreciable. To calculate the scales of $u$ and $w$ and to transform the correlograms to spectrum functions, it was necessary to make some assumption about the form of the curves as $x \rightarrow \infty$. It seemed reasonable to solve this problem by fitting exponential functions to the 'tails' of the curves; this was done in the case of $r_{u}(x)$ for $x>4$ meters and of $r_{w}(x)$ for $x>3$ meters.

Autocorrelation curves were obtained similarly from the other groups of records, and the

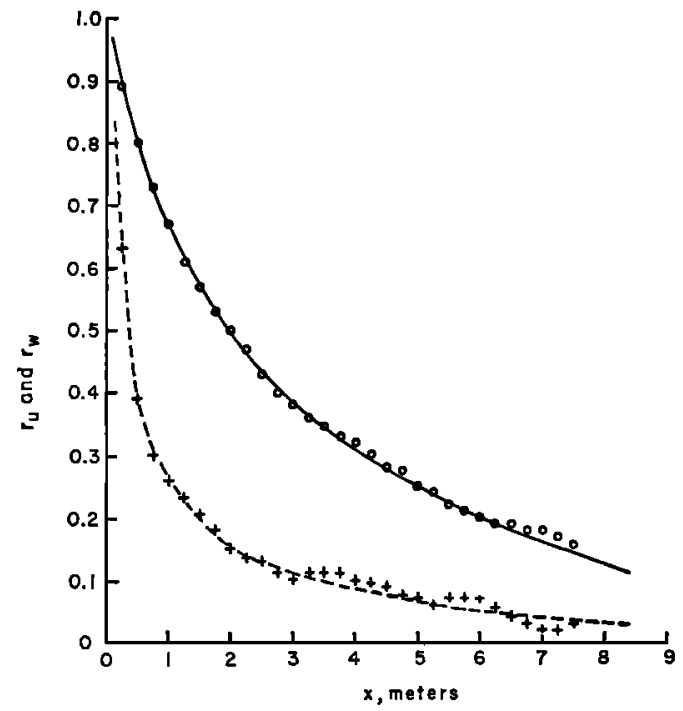

Fig. 1. Group of $6 u, w$ records at $75 \mathrm{~cm}$. Autocorrelations: open circles, $r_{u}(x)$; crosses, $r_{w}(x)$.

spectrum function $F(v)$ of $u$ and $w$ was derived in terms of the wave number $v$.

The spectrum of the transverse component $v$ was derived in the same way from the $u, v$ records. Figure 2(a) shows the spectra of the

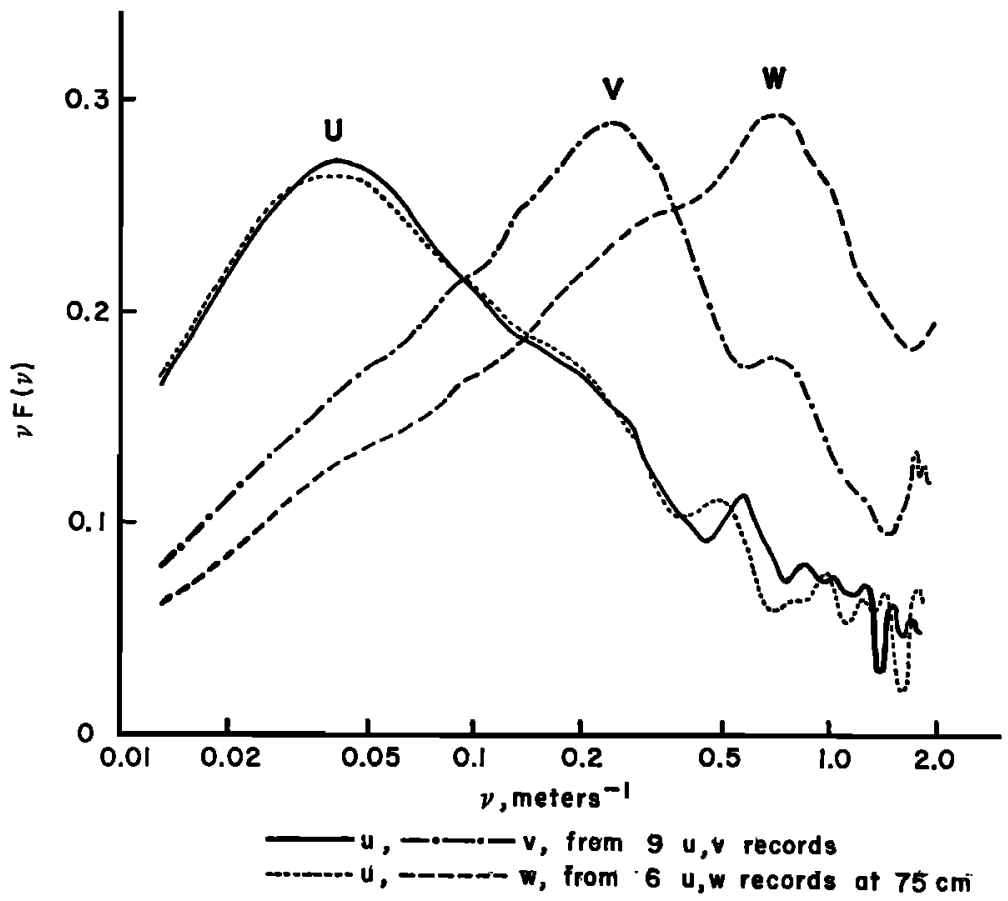

Fig. 2(a). Spectra of $u, v$, and $w$. 


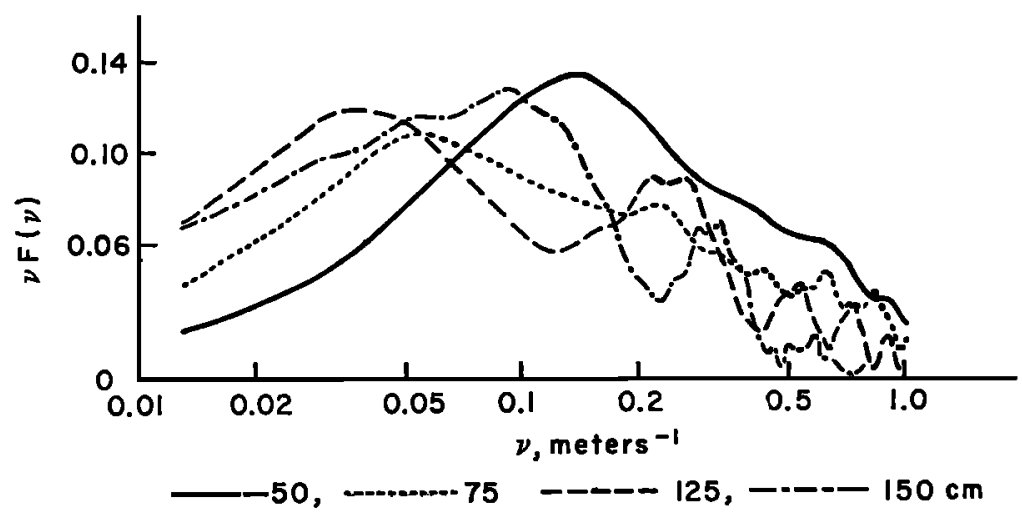

Fig. 2(b). Spectra of $[u w]$.

three components, $u, v$, and $w$, under comparable conditions. The two spectrum curves for $u$, obtained from the group of $u, v$ records and the group of $u, w$ records, respectively, are remarkably similar. The peak values of $v F(v)$ for the three components occur at $v=0.04 \mathrm{~m}^{-1}$ for $u, 0.25 \mathrm{~m}^{-1}$ for $v$, and $0.7 \mathrm{~m}^{-1}$ for $w$. The peak for $w$ corresponds to the value of $\nu \mathrm{z}=$ 0.5 , whereas in the lowest few meters of the atmosphere Panofsky and Deland [1959] found the peak at $\boldsymbol{\nu} z=0.2$ approximately.

The spectrum of the $u w$ product, i.e. of the Reynolds stress, is shown in Figure $2(b)$ for groups of records at each of the four heights. At each height the peak occurs at a value of $\nu$ intermediate between those of the $u$ and $w$ spectra, but closer to that of $u$. At $50 \mathrm{~cm}$ the peak is at $\nu=0.13 \mathrm{~m}^{-1}$ approximately, and at the other heights $\nu=0.06 \mathrm{~m}^{-1}$ approximately. In the atmosphere, Priestley [1959] has interpreted the data as indicating that the limiting wave number, above which the turbulence is isotropic and cannot contribute to the eddy stress, is given by $\nu z=0.6$ approximately. The curves in this figure suggest that fluctuations corresponding to $\nu z>0.6$ contribute little to the $u w$ spectrum in this case also.

$u_{1}, u_{2}$ and $w_{1}, w_{2}$ records. Turning now to records of the fluctuations in the $u$ component at two heights, Figure 3 shows the cross correlation $r_{u_{1} u_{0}}$ for various time lags. The mean curve for two groups each of five records with separations of $50 \mathrm{~cm}$ and $75 \mathrm{~cm}$ are shown. In each case the maximum correlation occurred not with zero lag but with $u_{1}$ at the upper level taken earlier than $u_{2}$ at the lower level. The

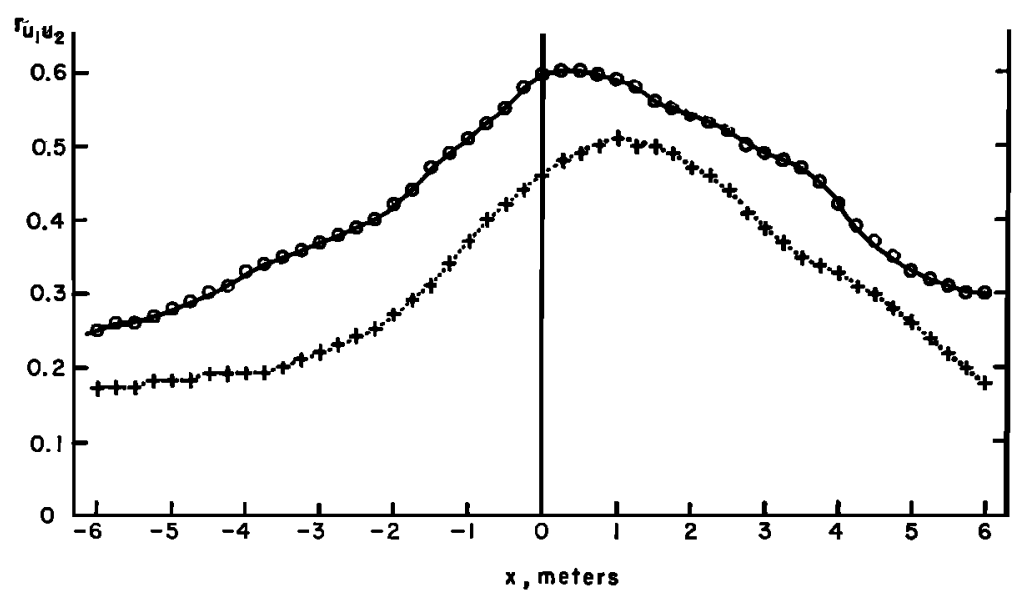

Fig. 3. Groups of $u_{1}, u_{2}$ records: cross correlations. Vertical spacing: open circles, $50 \mathrm{~cm}$; crosses, $75 \mathrm{~cm}$. 


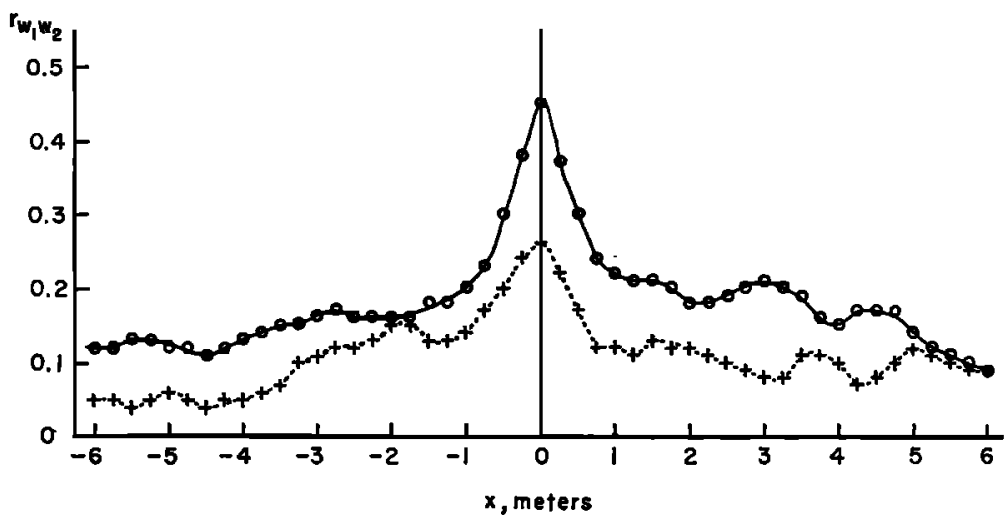

Fig. 4. Groups of $w_{1}, w_{2}$ records: cross correlations. Vertical spacing: open circles, $50 \mathrm{~cm}$; crosses, $75 \mathrm{~cm}$.

effect was more pronounced with a separation of $75 \mathrm{~cm}$, the maximum correlation occurring at a lag equivalent to $x=U_{\tau}=1.0$ meter. A similar effect was observed by Favre, Gaviglio, and Dumas [1957] in wind-tunnel experiments on boundary-layer turbulence and by Taylor [1958] in temperature fluctuations in the atmosphere.

Groups of records of the $w$ fluctuations at two heights have been analyzed in the same way. Figure 4 shows the cross-correlation curves for vertical spacings of 50 and $75 \mathrm{~cm}$. The curves are approximately symmetrical, with the maximum correlation $r_{w, w}$, occurring at zero lag, and there is no indication of the type of asymmetry found from the $u_{1}, u_{2}$ records. of records has fallen to the same value. Thus $\Delta z / x$ may be taken as an estimate of the ratio of the vertical scale of the $u$ fluctuations to the horizontal scale; this ratio is approximately $1 / 3$.

The last line of this table gives the mean results from two records of the $u$ fluctuations at two points separated horizontally, transverse to the mean flow. By a similar interpretation, the lateral scale of $u$ is estimated to be less than $1 / 4$ of the longitudinal scale. From only two records, however, we can deduce no more than that the lateral scale appears to be comparable with the vertical scale rather than with the scale in the direction of the mean flow.

The result that the vertical and lateral scales

TABLE 3. Mean Values for Groups of $u_{1}, u_{2}$ Records

\begin{tabular}{|c|c|c|c|c|c|c|}
\hline $\begin{array}{l}\text { Separation, } \\
\text { cm }\end{array}$ & $\begin{array}{l}\text { No. of } \\
\text { Records }\end{array}$ & $\begin{array}{c}\text { Range of } \\
U, \\
\mathrm{~cm} / \mathrm{sec}\end{array}$ & $\frac{\left[u_{1}^{2}\right]^{1 / 2}}{\left[u_{2}{ }^{2}\right]^{1 / 2}}$ & $\begin{array}{r}r_{u_{1} u_{2},} \\
x=0\end{array}$ & $\begin{array}{c}x \text { for } \\
r_{u}(x)=r_{u_{1} u_{2}}, \\
\mathrm{~cm}\end{array}$ & $\frac{L_{u z}}{L_{u x}}$ \\
\hline \multicolumn{7}{|l|}{ Vertical } \\
\hline & 5 & $22-48$ & 0.88 & 0.60 & 140 & 0.36 \\
\hline 75 & 5 & $30-39$ & 0.85 & 0.47 & 230 & 0.33 \\
\hline \multicolumn{7}{|l|}{$\begin{array}{c}100 \\
\text { Horizontal }\end{array}$} \\
\hline & & & & & & $L_{u y} / L_{u x}$ \\
\hline 65 & 2 & $35-39$ & 1.00 & 0.53 & 300 & 0.22 \\
\hline
\end{tabular}

The mean values of the correlation between simultaneous values of $u_{1}$ and $u_{2}$ for given vertical separations $\Delta z$ are shown in Table 3 (column 5). Column 6 gives the value of $x$ for which the autocorrelation curve for the same group of the $u$ component are considerably less than the longitudinal scale is in agreement with the data reported by Townsend [1956] for the constant stress layer in boundary-layer flow.

Table 4 gives mean values for the records of 
K. F. BOWDEN

TABLE 4. Mean Values for Groups of $w_{1}, w_{2}$ Records

\begin{tabular}{|c|c|c|c|c|c|c|}
\hline $\begin{array}{c}\text { Vertical } \\
\text { Separation, } \\
\text { cm }\end{array}$ & $\begin{array}{l}\text { No. of } \\
\text { Records }\end{array}$ & $\begin{array}{c}\text { Range of } \\
U, \\
\mathrm{~cm} / \mathrm{sec}\end{array}$ & $\frac{\left[w_{1}^{2}\right]^{1 / 2}}{\left[w_{2}^{2}\right]^{1 / 2}}$ & $r_{w_{1} w}$ & $\begin{array}{c}x \text { for } \\
r_{w}(x)=r_{w_{1} w_{2}} \\
\mathrm{~cm}\end{array}$ & $\frac{L_{w z}}{L_{w x}}$ \\
\hline $\begin{array}{c}50 \\
75 \\
100 / 125\end{array}$ & $\begin{array}{l}4 \\
5 \\
3\end{array}$ & $\begin{array}{l}22-41 \\
29-39 \\
25-32\end{array}$ & $\begin{array}{l}0.83 \\
0.82 \\
0.91\end{array}$ & $\begin{array}{l}0.45 \\
0.26 \\
0.17\end{array}$ & $\begin{array}{r}50 \\
100 \\
125\end{array}$ & $\begin{array}{l}1.0 \\
0.75 \\
0.93\end{array}$ \\
\hline
\end{tabular}

the $w$ fluctuations at two heights in the same way. It is seen that the correlation $r_{w_{1} w}$, decreases considerably more rapidly with increasing separation than $r_{u_{1} u_{2}}$ does. Estimating the ratio of the vertical and horizontal scales of $w$ indicates, however, that the vertical scale is not significantly less than the longitudinal scale, in contrast to the result for the $u$ component of the fluctuations.

Acknowledgments. I wish to acknowledge the assistance of my colleague, Mr. Fairbairn, who has worked with me throughout this investigation, and of Dr. Young, the Director of the Computer Laboratory at the University of Liverpool, and his staff, who have given much helpful advice in the use of the electronic computer.

\section{References}

Bowden, K. F., and L. A. Fairbairn, Proc. Roy. Soc. London, A, 237, 422, 1956.

Favre, A. J., J. J. Gaviglio, and R. Dumas, $J$. Fluid Mech., 2, 313, 1957.

Panofsky, H. A., and R. J. Deland, Advances in Geophys., 6, 41, 1959.

Priestley, C. H. B., Advances in Geophys., 6. 97, 1959.

Taylor, R. J., Australian J. Phys., 11, 168, 1958.

Townsend, A. A., The Structure of Turbulent Shear Flow, Cambridge University Press, New York, 1956. 